\title{
GESTIÓN CLÍNICA Y CONFLICTO DE INTERESES*
}

\begin{abstract}
Armando Ortiz Pommier**
Resumen: Exponemos una definición de lo que entendemos por "conflicto de intereses" y la influencia que puede tener esta situación mal manejada sobre la relación médico-paciente. Se proporcionan antecedentes que permiten concluir que el conflicto fundamental en la relación clínica es la llamada "doble agencia del médico". La bibliografía última en el tema se ha desatado como consecuencia del sistema conocido en Estados Unidos con el nombre de "Managed Care". Esa expresión se ha traducido de varios modos: en nuestro medio se conoce como "Gestión Clínica". La expresión norteamericana es muy precisa, porque en sus dos palabras quiere significar las dos funciones fundamentales del clínico: de una parte, su obligación de "care" y, por tanto, de buscar lo mejor para su paciente; de otra, su condición de "manager", es decir, de gestor de recursos, y la necesidad de que mire por el control del gasto. Este es el tema de la llamada "doble agencia del médico". Con este nuevo escenario va a cambiar radicalmente el futuro de la profesión médica, en cuya construcción nos parece que los profesionales tienen una responsabilidad indelegable. Estamos a las puertas de un cambio de paradigma en la forma de ejercer la medicina, lo que de todos modos significará un cambio en el contrato social de nuestra profesión.
\end{abstract}

Palabras clave: conflicto de intereses, doble agencia, ética, gestión clínica

\section{MANAGED CARE AND CONFLICTS OF INTEREST}

\begin{abstract}
We want to define what we understand as a conflict of interests and its influence upon the relationship doctor-patient when ill managed. We offer antecedents that allow us to infer that the fundamental conflict in the clinical relationship is the so called "doctor's double agency". Bibliography on the subject has increased as a consequence of the system known in the United States as "Managed Care". This expression is very precise because its two words imply the two fundamental definitions of a clinician: on one hand, his obligation to "care" and, therefore, to do his best for his patient; on the other hand, his condition of "manager", that is to say, the manager of resources and his need to look over the financial control. This is the subject of the so called "doctor's double agency". This new scenery will change the future of the medical profession and we think doctors have an undeniable responsibility. We are at the verge of a paradigmatic change in medical practice which will also mean a change in the social contract of our profession.
\end{abstract}

Key words: conflict of interests, double agency, ethics, clinical management

\section{GESTÃO CLÍNICA E CONFLITO DE INTERESSES}

Resumo: Expomos uma definição do que entendemos por "conflito de interesses" e a influência que pode ter esta situação mal administrada sobre a relação médico-paciente. São proporcionados antecedentes que permitem concluir que o conflito fundamental na relação clínica é a chamada "dupla militância do médico". Foi desatada a última bibliografia do tema como consequência do sistema conhecido nos Estados Unidos pelo nome de "Managed Care". Essa expressão foi traduzida de vários modos: em nosso meio se conhece como "Gestão Clínica". A expressão norte-americana é muito precisa, porque suas duas palavras querem significar as duas funções fundamentais do clínico: de uma parte, a obrigação de "care" e, portanto, de buscar o melhor para seu paciente; de outra, a condição de "manager", quer dizer, de gestor de recursos e da necessidade de que mire no controle do gasto. Este é o tema da chamada "dupla militância do médico". Este novo cenário vai mudar radicalmente o futuro da profissão médica, em cuja construçáo, segundo nos parece, os profissionais têm uma responsabilidade indelegável. Estamos às portas de uma mudança de paradigma na forma de exercer a medicina que, de todos os modos, significará uma mudança no contrato social de nossa profissão.

Palavras-chave: conflito de interesses, dupla militância, ética, gestão clínica

* Este artículo está basado en una publicación previa del autor (Ortiz A. Introducción al conflicto de intereses. Revista chilena de neuro-psiquiatría 2004, 42(1): 29-36).

** Médico neurocirujano, Magíster en Bioética. Departamento de Bioética y Humanidades Médicas, Universidad de Chile. Chile

Correspondencia: armando.ortiz@vtr.net 


\section{Introducción}

Si quisiéramos establecer cuál ha sido el modelo médico más reverenciado y alabado en el mundo occidental, no podríamos ignorar que el mundo de la salud ha asumido como propio a lo largo de los siglos el modelo de la medicina hipocrática -o de los hipocráticos como otros prefieren denominarlos. Nuestra medicina, como saber técnico y científico, ha tenido una conexión constante e inalterada con el compromiso ético que se expresa en el Juramento Hipocrático, sin duda uno de los documentos de mayor influencia y vigencia a lo largo de toda la historia de la medicina occidental. De allí toma como principio supremo de la actividad médica o de salud el de "favorecer y no perjudicar", que el último tiempo ha sido rectificado en algún tópico como "primero no hacer daño", acepción que dista mucho de ser correcta: el no hacer daño siempre fue subsidiario de hacer el bien, al menos así se entiende el precepto presente en el libro "Epidemias I" del Corpus Hippocraticum. Con el tiempo, este principio hipocrático derivó en los llamados principios de "beneficencia" y de "no-maleficencia", según los cuales, frente a un conflicto, es preciso priorizar como criterio de decisión el bien del paciente $\mathrm{o}$, al menos, no dañarle.

El problema es que durante siglos se entendió que es el médico quién debe discernir cuál es el bien del paciente, con lo cual el principio de beneficencia configuró el paradigma tradicional del paternalismo médico, que se puede interpretar en una de sus formas extremas como el rechazo, por el propio bien del paciente, de sus deseos y opciones, aun tratándose de una persona con información y capacidad adecuada de decisión. El imperio de esta forma de concebir la práctica clínica ha sido tal que ni siquiera el impulso emancipador que la Ilustración trajo a la vida política cambió el estilo de esa práctica, de modo que fue el principio de beneficencia, interpretado por los profesionales, el que comandó durante muchísimos siglos el modo de entender la toma de decisiones en la práctica de la medicina.

Ahora bien, lo ocurrido a lo largo del siglo XX obliga a preguntarse si ese modo de entender la práctica médica y esa actitud profesional de la ética hipocrática continúan vigentes y continuarán en el futuro; o si, por el contrario, el principio de beneficencia viene sufriendo una serie de percances que amenazan con destronarlo o al menos obligarlo a compartir el sitial que ocupa. Hay la sensación de que se terminará entendiendo que el "bien del paciente" -al que es preciso "favorecer y no perjudicar" - debe ser interpretado de otra manera. En la misma escalada, hay otros dos principios, que también hacen su esfuerzo por situarse en el podio de la consideración (los de autonomía y justicia), porque otros actores pugnan por ascender a ese sitial: los pacientes y los gerentes o directivos de las instituciones de salud.

La irrupción de la autonomía del paciente fue inobjetable luego de los escándalos en la experimentación médica, especialmente en la Alemania nazi, dando nacimiento como consecuencia de ello al Código de Nuremberg, en 1947. Este movimiento -que ha dado por llamarse el movimiento de emancipación de los pacientes- ha traído entre otras cosas la doctrina del consentimiento informado, no sólo en su forma de derecho propio del sujeto, sino también como extensión en la representación, incluso más allá de la condición de incapacidad, a través de los modelos de testamentos vitales o directrices previas. El principio hipocrático de beneficencia ha de complementarse con la noción que el paciente tiene de su propio bien. Lo que caracteriza a la ética médica de las décadas del setenta y el ochenta es la incorporación del principio de autonomía a la hora de tomar las decisiones. Esto quiere decir que las opciones personales del paciente deben ser atendidas y, en caso de conflicto entre los principios en juego (beneficencia por parte del médico y autonomía por parte del paciente), debe primar el de autonomía. "A la hora de establecer prioridades, las preferencias del paciente son la categoría ética de más peso en el encuentro entre el médico y el paciente".

En la década de los ochenta se abre un nuevo frente en contra del principio hipocrático. Ahora ataca no la autonomía del paciente, sino la economía. Ataque que se desata desde el momento en que la medicina pasa a ser asunto público. En concreto, cuando los Estados sociales, en forma de Estados de bienestar, incrementan los gastos en salud de modo acelerado y alarmante. $\mathrm{Al}$ convertirse la salud en un problema público, la atención pasó a ser asunto de justicia social en lo que toca a la distribución y asignación de recursos en salud. A partir de ese momento se empieza a revisar la racionalidad de las políticas de bienestar y, entre ellas, las de salud, sobre todo por la necesidad de controlar el gasto.

La aplicación a la salud de teorías de la justicia (como la que Norman Daniels hace de la teoría de John Rawls), entendiendo el derecho a la asistencia en salud como un "bien primario", subsidiario del principio rawlsiano 
de "igualdad de oportunidades", exige determinar las necesidades de asistencia médica, ya que es imposible atenderlas a todas. De entre las múltiples respuestas destaca la del "mínimo decente", el intento de determinar unos mínimos de justicia moralmente exigibles que garanticen la atención en salud básica igual para todos. Pero estas exigencias chocan con la escasez de recursos, obligando a enfrentarse a los problemas de financiamiento y gestión de los recursos de salud.

El crecimiento explosivo e ininterrumpido de los costos -otra característica de la medicina actual- y la imposibilidad de contenerlos en una sociedad consumista, hicieron pensar que la situación sería insostenible. Los economistas acusaron a los políticos y a los médicos de irresponsabilidad en la gestión de los recursos sanitarios y nació la "economía de la salud", que se propuso introducir la racionalidad económica en este campo, ocupado tradicionalmente por el compromiso tradicional del médico, orientado por el principio de beneficencia. Los problemas de financiamiento y gestión irrumpieron con fuerza en el mundo de la salud y la racionalidad económica se convirtió en un ingrediente de la preocupación bioética, precisamente por razones de justicia.

Tras una etapa de expansión y desarrollo acelerado del gasto en salud (años setenta y ochenta) y otra de contención o reducción de costos (años ochenta), fue preciso hacer frente a una nueva situación en la que primó la evaluación de las prácticas clínicas y la responsabilidad empresarial y profesional, especialmente porque la gestión del bien "salud" y la relación entre los profesionales de la salud y los pacientes se vive cada vez más en un medio hospitalario, administrado por alguna entidad estatal o bien convertido en una empresa de salud. En cualquier caso, esas instituciones deben estar estrechamente relacionadas entre sí, porque lo verdaderamente decisivo es enfrentarse a la nueva situación.

Por ello, un aluvión de transformaciones sin precedentes en el entorno de la formación y el ejercicio de la práctica de la medicina desencadenó, a escala mundial, un intenso proceso de revisión de la autonomía y regulación de la profesión médica y sus relaciones con la sociedad y el Estado. El análisis de los factores explicativos de ese cambio y la discusión sobre la recomposición del equilibrio de poderes entre ciudadanos, políticos y médicos, así como la nueva naturaleza del "contrato social" de la profesión con la sociedad, forman parte de la vanguardia de aportación crítica de grupos de pacientes, profesionales, expertos y académicos en los países más avanzados.

\section{El futuro de la profesión médica: un reto para los profesionales}

Intentar distinguir los futuros deseables y posibles, logrando ofrecer una visión de conjunto de los nuevos desafíos que enfrenta el profesional médico, y abrir el debate y el análisis con el fin de que puedan ofrecerse soluciones específicas al final de esta década debiera ser uno de los objetivos de los diferentes colectivos profesionales: sociedades científicas, asociaciones gremiales y otros.

Con el término "medicina gestionada" (Managed Care) se alude a los diferentes modos de introducir el mercado y la empresa en el mundo de la salud, lo cual puede ser necesario, pero siempre que queden claros los costos y a costa de qué se invierten, porque puede haber costos que rebasen todo precio y pongan en peligro justamente las metas que dan sentido a la actividad de la salud, valores éticos que marcan el límite de lo moralmente aceptable. Indudablemente, la economía y la gestión empresarial han tenido un papel relevante en el desarrollo de la medicina tal cual la conocemos hoy día, e igualmente indudable es que la justicia sanitaria exige la eficiencia; pero el giro favorable al mercado y la privatización para contener el gasto, tal como se ha producido, tiende a convertir la medicina en una mercancía y pone en peligro sus valores éticos sustanciales.

En este sentido, importa averiguar si las innovaciones de la gestión empresarial destruyen la relación fundamental de confianza entre el médico y el paciente. Puesto que si el médico se convierte en gestor de recursos y en controlador del gasto y si se establecen incentivos y sanciones proporcionales al ahorro o al gasto, cabe sospechar que el médico se verá obligado a actuar como un "agente doble", que mirará tanto por las necesidades del paciente como por reducir los gastos de salud.

Surgen así conflictos entre la medicina gestionada y las tradiciones éticas médicas, tanto hipocrática como liberal, que han puesto en primer lugar la atención al paciente, ya sea dando prioridad a su bien (desde el principio de beneficencia) o a su voluntad (desde el principio de autonomía). Si la medicina gestionada quiere actuar por razones de justicia, por razones éticas, tendrá que evitar destruir valores propios de las profe- 
siones de la salud, como es el caso de la confianza en las relaciones terapéuticas, puesto que al desvirtuarlas estará corrompiendo las relaciones profesionales, lo que irá en perjuicio de la propia asistencia y eficiencia. No todo consiste en reducir el gasto; para que la gestión sea legítima y justa es preciso saber por qué, cómo y para qué. Como nunca, o tal vez como siempre, nuestra profesión está inmersa en sus propias tribulaciones técnicas y éticas, humanas y científicas, a las que hay que sumarles todo este vendaval de cambios descritos.

\section{Algunas definiciones}

Conflicto de intereses: En su forma más original o primitiva, el conflicto es parte natural de las relaciones humanas, puesto que surge de las diferencias e incompatibilidad de intereses, percepciones u objetivos entre dos o más personas. En opinión de Thompson, se origina en aquellas circunstancias en que el juicio profesional en relación con su interés primario, como puede ser el bienestar del paciente para el clínico o la validez de la investigación para el investigador, o el interés educativo o asistencial, se ve influenciado indebidamente o en exceso por un interés secundario, como puede ser un provecho económico (beneficio financiero) o un afán de notoriedad, prestigio personal o el reconocimiento y promoción profesional(1).

El juicio profesional se refiere al proceso de ponderación y toma de decisiones sobre la base de la capacitación propia de su profesión. El interés primario viene determinado por los deberes profesionales, que en el caso del médico es el bienestar del paciente y en el caso del investigador la obtención de conocimiento válido generalizable(2). El interés secundario puede tenerlo el médico o el investigador en su actuación, y no tiene por qué ser ilegítimo en sí mismo, por ejemplo, avanzar en la carrera académica o, incluso, puede ser de índole altruista (conseguir fondos para ulteriores investigaciones sobre enfermedades "huérfanas")(3). El problema es que estos intereses secundarios pueden adquirir un grado problemático de influencia en la toma de decisiones.

\section{¿Qué es necesario para que se dé un conflicto de} intereses?: En el medio legal anglosajón, el término "conflicto de intereses" se utiliza primariamente en conexión con el fiduciario, aquel profesional que posee algún tipo de poder, basado en su capacitación especializada, que debe ser usado para el beneficio de otro. Una relación fiduciaria supone dependencia y confianza y está sujeta a los más altos estándares de conducta(4). En este sentido, la relación médica es un tipo de relación fiduciaria, en la cual el profesional médico ostenta un poder que deberá utilizar para promover el bienestar del paciente(5).

Para que exista un conflicto de intereses deberá existir un tipo de relación fiduciaria -como puede ser la relación médica, docente, investigadora- con un interés primario claramente definido que debe dirigir la toma de decisiones. Se establece el conflicto cuando en el proceso decisional entran a considerarse uno o varios intereses secundarios (prestigio personal, reconocimiento académico, promoción, incentivos económicos), que pueden determinar acciones alejadas o contrarias a los intereses profesionales primarios.

Ahora bien, estos intereses en conflicto nunca son equivalentes desde un punto de vista ético, ya que la relación profesional entre el médico y el enfermo o entre el investigador médico y los enfermos está fundamentada (tiene su razón de ser) precisamente en sus intereses primarios(1).

\section{¿Por qué es problemático que se dé un conflicto de intereses?: Todos tenemos intereses, aunque sea en diferente grado u objeto, y casi no hay actividad hu- mana que en el fondo no pretenda obtener algún tipo de beneficio. La presencia de un conflicto de intereses no supone por sí misma que se producirá un desenlace incorrecto éticamente, pero es evidente que incrementa su posibilidad. Además, que la sociedad constate que no es correctamente manejado mina la confianza en la asistencia médica y en la investigación clínica, y evidentemente la viabilidad futura de ambas $(6,7)$.}

\section{¿Qué pasa con la relación clínica?}

Dejando de lado los cambios históricos en la relación médico-paciente y sobre todo la actual incorporación de múltiples actores a la misma, en una sociedad democrática en la que todos los individuos son, mientras no se demuestre lo contrario, agentes morales autónomos, capacitados para tomar decisiones, con criterios distintos sobre lo que es y lo que no es malo, la relación médica, en tanto que relación interpersonal, es esencialmente conflictiva(8).

Ya es un tópico lo que ha señalado muchas veces Diego Gracia: "Hay muchas razones para pensar que la medicina ha evolucionado en estos últimos veinticinco años más que en cualquier otro período de su historia"(9). 
La irrupción en el mundo sanitario de muchos otros actores -administradores, auxiliares de servicio, otros profesionales de áreas de la salud, instituciones, entre otros- no sólo ha generado cambios en la relación clínica, sino que también se han debilitado más las reglas tradicionales de fidelidad. En particular, las empresas financiadoras y los proveedores institucionales han impuesto limitaciones a las decisiones médicas sobre el diagnóstico y las intervenciones terapéuticas. Estos intereses económicos forman parte de la vida diaria de los médicos y formarán una parte mayor en el futuro. La nueva situación establecerá que los médicos deberán mantener lealtad con diversas instituciones, así como con los pacientes (10).

Frente a un nuevo entorno laboral, caracterizado por el aumento sostenido del costo de la salud, se han establecido nuevos mecanismos de control, incluyendo pago por adelantado, grupos relacionados con el diagnóstico, revisiones de utilización, acuerdos de proveedor preferido y diversas formas de medicina gestionada ${ }^{1}(10)$. Estos mecanismos a menudo condicionan y reducen la fidelidad del médico hacia el paciente, a través de una mezcla de incentivos y medidas disuasorias, que a veces ponen el interés del médico en pugna con el mejor interés del paciente, produciendo serios conflictos éticos y de fidelidad(2). El paciente está en una posición muy diferente cuando el médico tiene incentivos para restringir los tratamientos necesarios que cuando los tiene para ofrecer los tratamientos innecesarios. En la última situación, los pacientes pueden obtener otra opinión; en la primera, pueden ignorar la necesidad de un tratamiento porque nadie se lo ha recomendado(10).

¿Cuál será el principal conflicto de intereses que enfrentarán los futuros médicos o los que ejercen actualmente la profesión? La bibliografía última se ha desatado como consecuencia del sistema conocido en Norteamérica con el nombre de "Managed Care". Esa expresión se ha traducido de varios modos al espańol, como "cuidado dirigido" en Puerto Rico o como "gestión clínica" en España. La expresión norteamericana es muy precisa, porque en sus dos palabras quiere significar las dos funciones fundamentales del clínico: de una parte, su obligación de "care" y por tanto de buscar lo mejor

El Managed Care está introduciendo gran cantidad de conceptos económicos en la jerga ética, de realidades de implantación todavía incierta en nuestro sistema sanitario, como las enumeradas: prospective payment, diagnosis related group, utilization review, preferred provider arrangements, fee-for-service, self-referral, etc. para su paciente; de otra, su condición de "manager", es decir, de gestor de recursos, y la necesidad de que atienda el control del gasto. Este es el tema de la llamada "doble agencia del médico", el conflicto de intereses fundamental. Lo cual no quiere decir que no puedan y deban armonizarse ambas dimensiones del acto clínico. Ése es uno de los objetivos de la bioética: ayudar a resolver ese tipo de conflictos.

\section{Ser médico o hacer de médico en el siglo XXI. El problema de la "doble agencia"}

En el ambiente sanitario -en términos cotidianos, "en una cafetería de hospital”-, los médicos declaramos percibir a los economistas y sus intereses liberales como los principales responsables de nuestras desgracias y desventuras, explicación en principio imprecisa. Intentar acercar las perspectivas del economista y del médico, perniciosamente enfrentadas en el contexto actual, no es tarea fácil, pero coincidamos en que es mucho más fructífero buscar los puntos comunes que ahondar sus diferencias(11). Y en este enfoque hay al menos dos aspectos relevantes: por una parte, el problema de la doble lealtad o doble agencia, generado por la presencia de terceros pagadores, y de cómo los conflictos de intereses que de ello surgen debilitan el modelo hipocrático al enfrentarlo con la ética corporativa; por otra, la necesidad de que el médico asuma su responsabilidad en la distribución de recursos, como estrategia para conservar su autonomía, pero, a la vez, la necesidad de renegociar el contrato entre la profesión y la sociedad. Y si no son los médicos, ¿quiénes tomarán el liderazgo para que los principios fundamentales de la profesión no se vean aún más afectados?

El modelo tradicional de práctica médica que prevaleció por veinticinco siglos era el de un paciente que acudía a su médico para que éste actuara como su agente y tomara decisiones en su beneficio, utilizando para ello un conocimiento avanzado pero con total autonomía. Entre estos dos mediaba el honorario, una forma de honrar a quien ponía su conocimiento al servicio del paciente. Durante la segunda mitad del siglo XX empezó la carrera tecnológica que desbordó a todos los países, incluidos los más ricos y poderosos, y por tanto la capacidad de los hogares de enfrentar por sus propios medios los costos de un evento de enfermedad o accidente. Aparecieron los seguros como un mecanismo de protección frente a la probabilidad de ruina financiera y con éstos desapareció de la relación tradicional el 
problema de la capacidad de pago. Los seguros no hicieron más que acelerar el proceso de encarecimiento de los servicios de salud, pues ya no había limitantes en la capacidad de pago por parte del paciente, generándose los conocidos problemas del riesgo moral y el facilitamiento de la inducción de la demanda.

Entre otras, surge la dicotomía que en su aspecto fundamental consiste en que el médico recibe una remuneración adicional que no es conocida por el paciente. Con frecuencia, la dicotomía es injusta para el enfermo, que a menudo sufre una agresión económica o no recibe el tratamiento más adecuado para su condición. También resulta perjudicial para los médicos que rechazan la dicotomía ya que quedan condenados a una situación de inferioridad económica en comparación con aquellos que sí la aceptan. En los lugares en que esta práctica se ha generalizado, el médico invariablemente pierde su independencia de juicio y se refieren los pacientes no al colega más competente para ese caso o se sobreutilizan procedimientos o intervenciones. Siempre esta situación es descubierta, con el consiguiente descrédito no sólo para los médicos implicados sino que para toda la profesión(2).

Puesto que esto no puede seguir indefinidamente así, aparecen los planes de salud integrales, con un control más estricto del gasto médico, control que sólo logran los aseguradores mediante incentivos financieros y no financieros para que el médico racionalice el uso de los recursos de que dispone.

Cualquier mecanismo de distribución de recursos genera el problema de la doble lealtad. La única posibilidad de recuperar la lealtad exclusiva del médico con el paciente es volviendo a la responsabilidad del paciente por la totalidad del costo de la atención, y es obvio que eso no va a ocurrir. La doble lealtad no es pues un problema generado exclusivamente por el asegurador, sino también por cualquier mecanismo de dispersión de riesgos y costos financieros, cualquiera sea su escala.

Según lo anterior, el problema de la doble lealtad no sería un problema moralmente cuestionable, sino una consecuencia obligada de la evolución de la prestación de servicios de salud. Lo que frecuentemente se critica de este problema es más bien algo que se oculta tras él: el fin de lucro, que se hizo evidente con la entrada de la medicina prepagada o el sistema "Isapre ${ }^{2 "}$ en los

2 Instituciones de Salud Previsional en Chile. ochenta, y pareciera que pronto en el sistema público de salud. Si el fin de lucro per se no es lo cuestionable, podría decirse que la ética corporativa de estas organizaciones es un instrumento de la ética distributiva para lograr su objetivo de eficiencia en el gasto en salud.

Es evidente e inevitable que la práctica médica actual esté condicionada por esta doble lealtad, puesto que el médico es agente simultáneo de dos principales: el paciente y el tercer pagador. Pero más que el tercer pagador, el verdadero segundo principal del médico es el grupo de personas asociadas en el fondo de seguros; el tercer pagador no es más que un mediador de esa obligación, y que además cobra por correr un riesgo, al menos en el contexto nuestro.

Los conflictos de intereses generados por la doble lealtad no son, por sí mismos, más problemáticos que otros tipos de conflictos de intereses, presentes desde que apareció la remuneración al profesional por sus servicios. El problema fundamental con estos conflictos radica en su intensidad(1): cuando el interés secundario es $\tan$ fuerte que compromete severamente el interés primario es evidente que se ha invertido la obligación del agente para su principal hacia su beneficio; pero esto puede suceder en cualquier contexto y no es exclusivo de la intermediación con fin de lucro. Por lo tanto, nuestra preocupación debe orientarse a controlar la intensidad de los conflictos de intereses, más que el fin de lucro o la simple presencia de tales conflictos.

En síntesis, respecto de este segundo punto, los recursos que el médico utiliza para tratar a su paciente no le pertenecen a éste ni a aquél, sino al grupo de personas que han optado por un esquema cooperativo de protección financiera. El hecho de que la capacidad de pago del paciente desaparezca del acto médico no implica ignorar el costo de oportunidad de las decisiones clínicas; por el contrario, implica una responsabilidad mayor frente a la colectividad, lo cual inevitablemente crea la doble lealtad en el médico: frente a su paciente y frente a los demás beneficiarios de los recursos disponibles(11).

No queda duda de que el modelo tradicional de práctica médica, basado en la ética hipocrática y en la autonomía propia del profesionalismo, está actualmente y de manera irreversible condicionado por la doble lealtad del médico. Este condicionamiento ha sido visto como una amenaza al profesionalismo, postura que considero es la mayoritariamente adoptada por los médicos nostálgicos. Creemos que ello no es necesariamente cierto 
y que, por el contrario, la profesión debe asumir los nuevos retos que la doble lealtad supone para conservar ese preciado don del profesionalismo.

Si el médico es quien finalmente determina cómo se utilizan las cuatro quintas partes de los presupuestos para salud y si los individuos ya no llegan al médico por su propia cuenta sino amparados por un tercer pagador, es claro que el médico tiene hoy la obligación de hacer una utilización racional de los recursos. Si no lo hace, los terceros pagadores lo harán por él: lo presionarán a seguir protocolos, lo someterán a revisión por pares o a segundas opiniones, o finalmente lo obligarán a asumir riesgo financiero bajo contratos de capacitación u otros incentivos monetarios.

Pero no se trata de que el médico se obstine en defender una posición que a todas luces en el contexto actual es insostenible. En sana lógica, si el médico extiende su principio de justicia a su obligación como distribuidor de recursos está cumpliendo con una obligación que hoy no puede eludir, ejerciendo en el micronivel lo que el formulador de políticas ejerce a diario cuando toma decisiones de distribución de recursos en el macronivel. Pero ello supone inevitablemente tomar decisiones trágicas: restringir el acceso de unos para permitir un mejor acceso a otros y de este modo contribuir a que los recursos sean usados de manera eficiente, sin necesidad de un supervisor que coarta su autonomía sobre la base de desautorizaciones y órdenes incuestionables.

Reaparece aquí el concepto de "autonomía” como eje del profesionalismo. Evidentemente, en el contexto de restricciones que he descrito, qué mejor manera de recuperar la autonomía profesional que asumiendo de manera racional la responsabilidad en la distribución de recursos. Si el médico no quiere verse vigilado por un extraño, lo lógico es que haga por su propia cuenta aquello por lo cual hoy es vigilado. Pero este cambio de paradigma supone una renegociación del contrato social representado por el juramento hipocrático. El rediseño de las obligaciones del médico frente a la sociedad requiere un proceso en el cual participen los beneficiarios de las decisiones del médico, es decir, los pacientes actuales y futuros. La sociedad debe entender que si espera que sus médicos siempre busquen el beneficio del paciente sin importar el costo, estarán generando un resultado subóptimo en la utilización de los recursos para salud. Por esto, la renegociación del pacto social debe comprometer tanto a los profesionales como a la sociedad en general, mediante un proceso democrático, deliberativo y abierto que considere objetivamente las restricciones presupuestales, la disponibilidad de pagar de los asociados y la nueva responsabilidad del profesional en el racionamiento.

La renegociación del contrato social no es una claudicación de la ética hipocrática ante la ética distributiva; es más bien una versión ampliada del compromiso del médico con su paciente, que reconoce la responsabilidad del profesional en la adecuada utilización de los recursos de la sociedad. Es el camino lógico y sensato hacia la preservación del profesionalismo, que hace irrelevante la amenaza del control externo sobre la autonomía y que, del mismo modo, permite construir entre los dos una relación en la que la profesión sale mejor librada. A la vez, es consecuente con la búsqueda del equilibrio entre equidad y eficiencia y con la prestación de servicios de alta calidad. En síntesis, la obligación del profesional sigue siendo frente a su paciente, pero en conexión con los demás pacientes.

\section{Comentarios}

¿Cómo afrontar el reto de mejorar sin renunciar a lo que hemos conseguido? ¿Cómo debe evolucionar la profesión médica para sobrevivir y para que contribuya a la sostenibilidad de los logros alcanzados haciendo posibles nuevos hitos? ¿Cómo construir las visiones de futuro deseables y también posibles? Nadie duda de que el milenio ha comenzado con una crisis de los sistemas de salud, que no coge a los profesionales desprevenidos aunque sí erosionados, y a los ciudadanos irritados e insatisfechos.

Mucho es lo que se habla en los últimos tiempos de la pérdida de confianza del público en la figura del médico y en general en la profesión médica. ¿Qué ha sucedido que se ha transformado en una paradoja? Pues, "mientras mayor ha sido el desarrollo de la tecnología y la capacidad de la medicina para resolver algunos problemas de salud, mayor es su situación de desprestigio". ¿Cuál es en realidad el verdadero privilegio que los médicos sienten que han perdido? Más allá de todas las posibles explicaciones, el privilegio perdido ha sido la confianza. Hay quienes sostienen que todavía la medicina es la profesión más valorada por la población en los países europeos y en muchos otros del mundo entero, y que por tanto la opinión negativa de los ciudadanos se dirige más al sistema que a los médicos, por no haber cumplido con el objetivo de colocar al paciente en el centro del sistema, burocratizar el sistema, favorecer la 
falta de información, la generación de listas de espera y la saturación de los servicios de urgencia. Pero, ¿acaso todo esto no recuerda con familiaridad el trabajo hospitalario actual?

Los expertos coinciden en que la relación médicopaciente será en el futuro el eje central del nuevo modelo organizativo. Una relación a la que, nos guste o no, los médicos deberán adaptarse, debiendo por tanto generar en los profesionales del futuro las actitudes que les permitan ejercer con un nuevo tipo de relación médico-enfermo más igualitaria, en la cual los pacientes son conscientes de su derecho a la información y a la autonomía y no aceptan de sus médicos actitudes paternalistas o autoritarias. Recabar el consentimiento informado de los pacientes como prueba de que se les ha informado y no como salvaguarda del médico deberá tender a ser una práctica cada vez más extendida.

El bioeticista Daniel Callahan(12) propone llevar a cabo un replanteamiento más radical, formulando nuevos ideales y direcciones. Si no se hace esto último, la medicina será: "económicamente insostenible, clínicamente confusa, socialmente frustrante y carente de dirección y propósito coherentes". El informe del Hastings Center(12) sobre Goals of Medicine sugiere que la medicina preste atención preferente a cuatro grandes objetivos: prevención de la enfermedad, promoción y mantenimiento de la salud; alivio del dolor y del sufrimiento humano causado por los padecimientos de orden interno; cuidado y curación de todos los que tienen padecimientos y cuidado de los que no pueden ser curados; evitación de las muertes prematuras y aspiración a una muerte en paz.

De tal manera, la crisis que enfrentarán los futuros profesionales se vincula más con una crisis de la medicina en sus propios fines y no sólo ni fundamentalmente en los medios que emplea. Hasta hora los problemas de la medicina actual han sido exclusivamente técnicos y organizativos y se han dejado de cuestionar los fines y los presupuestos más básicos. El giro de timón deberá orientarnos a sus verdaderos fines.

Es razonable esperar que el debate generado en este artículo nos ayude a entender algunos de los cambios que en los próximos años se producirán, tanto en las coordenadas organizativas -en cuya conformación las profesiones pueden intervenir en pie de igualdad con otros agentes del sistema y de la sociedad-como en la modernización y posicionamiento profesional, a cuya estrategia y definición los profesionales deben dar una respuesta específica e indelegable.

\section{Referencias}

1. Thompson DF. Understanding financial conflict of interest. N Engl J Med 1993; 329: 573-576.

2. Novoa F. Conflicto de intereses en la relación médico-paciente. Panorama Médico 1998; 34: 5-6.

3. Lemmens T, Singer PA. Bioethics for clinicians: 17. Conflict of interest in research, education and patient care. CMAJ 1998; 159: 960-965.

4. Rodwin MA. Strains in the fiduciary metaphor: divided physician loyalties and obligations in a changing health care system. Am J Law Med 1995; 21: 241-257.

5. Lo B. Resolving ethical dilemmas. A guide for clinicians. Philadelphia: Lippincott Williams and Wilkins; 2000.

6. Mastroianni A, Kahn J. Swinging on the pendulum. Shifting views of justice in human subjects research. Hastings Cent Rep 2001; 31: 21-28.

7. Ara JR. Manejo de los conflictos de intereses en la investigación médica. Visión desde la clínica. ICB digital, septiembre 2002. Disponible en http://www.icbdigital.org/icbdigital/pdf/resumen8.pdf

8. Gracia D. Fundamentos de bioética. Madrid: Eudema; 1989.

9. Gracia D. Bioética clínica. Bogotá: El Búho; 1998.

10. Beauchamp TL, Childress JF. Principios de ética biomédica. Barcelona: Masson; 1999.

11. Castańo RA. Medicina Ética y Reformas a la Salud. Marzo 2004. Disponible en http://www.encolombia.com/medicina/ academedicina/m-08RAbel.htm

12. Sánchez MA. Historia, teoría y método de la medicina: Introducción al pensamiento médico. Barcelona: Masson, 1998.

Recibido: 22 de septiembre de 2008

Aceptado: 28 de octubre de 2008 